\title{
EVOLUÇÃO DAS PESQUISAS SOBRE AS AMEAÇAS ÀS PEQUENAS ORGANIZAÇÕES
}

Ana Luisa Dal Belo Carneiro Leão ${ }^{1}$

Vânia Maria Jorge Nassif ${ }^{2}$

\footnotetext{
${ }^{1}$ Universidade Nove de Julho - UNINOVE

${ }^{2}$ Mestrado e Doutorado / Programa de Pós-Graduação em Administração / Universidade Nove de Julho
} 


\section{EVOLUÇÃO DAS PESQUISAS SOBRE AS AMEAÇAS ÀS PEQUENAS ORGANIZAÇÕES}

Resumo: Neste estudo, examinamos e damos sentido às pesquisas sobre as ameaças às pequenas organizações. Numa amostra de 953 artigos e 21.467 citações, realizamos análises de citações, cocitações e empregamos técnicas de análise fatorial. Os resultados permitiram identificar os trabalhos mais influentes sobre as ameaças às pequenas organizações e a variação da influência relativa destes estudos ao longo do tempo; o core list dos periódicos que publicam o tema; a estrutura intelectual da pesquisa com base em matrizes de cocitações; e a evolução do tema a partir de sete fluxos teóricos. Esse estudo oferece possibilidades de pesquisas futuras e contribui para o desenvolvimento das lacunas de pesquisas sobre os tipos de ameaças.

Palavras-chave: Tipos de ameaças. Bibliometria. Análise de citações. Análise de cocitação.

\section{Introdução}

Parece ser redundante afirmar a importância das pequenas organizações para o setor econômico e social de um país. Os motivos que levam às pequenas organizações ao sucesso (Sandberg \& Hofer, 1987; Trailer, Hill \& Murphy, 1996; Chen, Greene \& Crick, 1998; Simon, Horighton \& Aquino, 1998; Stewart et al., 1999; Aquino, 2000; De Brentani, 2001; Baker \& Sinkula 2009; Unger et al., 2011; Dickel, Hörisch \& Ritter, 2018; Tomenendal et al., 2018; Agbenyegah, 2018; Cox \& Nguyen, 2018; Gupta \& Mirchandani, 2018) e ao fracasso (Gibb, 1987; Duchesneau e Gartner, 1990; Bates, 1994; Gatewood, Shaver e Gartner, 1995; Trailer, Hill e Murphy, 1996; De Meza, Southey, 1996; Simon, Horighton \& Aquino, 2000; Huggins, 2000; De Brentani, 2001; Mendy, Hack-Polay, 2018; Van Tassel, 2018; Lin, Peña \& Chen, 2017; Du Plessis \& Marnewick, 2017) vem sendo amplamente estudado na literatura. Compreender as ameaças (Tsang 1994; Palech, Ray \& Bagby, 1995; Krueger, Rully \& Carsrud, 2000; Harris, Rae 2009; Ishaq, Mussain \& Whittan, 2010; Massey \& Pren 2012; Bloch 2016; Singer \& Minola, 2017; Lópes Lópes et al., 2017; Sarkar, 2017; Palanisamy, Rajendiran \& Prabu, 2017; Grabricler \& Grimm, 2017). Reconhecer essas ameaças e os comportamentos de enfrentamentos dos empreendedores, bem como as emoções envolvidas ao vivenciá-las, parece aumentar a probabilidade de sobrevivência das pequenas organizações. Mas como as pesquisas sobre ameaças têm evoluído ao longo dos anos?

Estudos envolvendo os tipos de ameaças enfrentados pelas organizações não são recentes. Na literatura é possível verificar que as pesquisas sobre o tema tiveram um grande impulso a partir do ano de 1990, em decorrência da globalização dos mercados, da necessidade das organizações enfrentarem as forças globais da concorrência e de se adaptarem para sobreviver no mercado competitivo (Hadjimanolis, 1999). Concomitantemente a essas forças globais, surge a preocupação dos pesquisadores para explicar quais são os fatores que contribuem para as organizações serem bem-sucedidas neste cenário, e aqueles que propiciam o seu crescimento e desenvolvimento (Yasuda, 2005). Esses estudos muitas vezes envolvem temas como obstáculos, barreiras, declínio organizacional, comportamentos de enfrentamento, fracasso, entre outros. $\mathrm{O}$ que se nota ao revisitar a literatura sobre o tema ameaças às pequenas organizações, é que as investigações com maior influência buscam compreender os tipos de ameaças enquanto problemas estratégicos e operacionais dominantes. Nessa lente teórica, os estudiosos relacionam os obstáculos, problemas, dificuldades e barreiras, com as etapas do
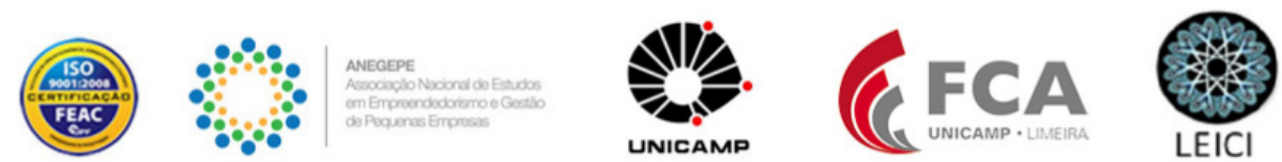
modelo de estágio de crescimento dos negócios (Miles \& Snow, 1978; Porter, 1980; Midgley, 1981; Block \& MacMillan, 1985; Kazanjian, 1988; Orser, Hogarth-Scott \& Riding, 2000; Mambula, 2002; Yasuda, 2005; Robson \& Obeng, 2008). Porém, novas correntes teóricas surgem, aumentando os desafios quanto à compreensão do tema no campo do empreendedorismo. Assim, este artigo tem por objetivo examinar e dar sentido às pesquisas sobre as ameaças às pequenas organizações, considerando que o mesmo possa contribuir, especialmente, por reconhecer o que vem sendo produzido e o que poderá ser investigado no futuro. Utilizamos as técnicas bibliométricas padrão em artigos publicados e recuperados da base de dados Scopus, WoS e Capes para a realização da presente pesquisa. Foram realizadas análises de frequências de citação e de fatores para derivar os subcampos das pesquisas sobre ameaças às pequenas organizações. As análises estruturais permitiram resumir as conquistas e, compreender como os esforços de pesquisa vêm evoluindo. Os resultados identificam os trabalhos mais influentes sobre os tipos de ameaças às pequenas organizações, bem como a variação da influência relativa destes estudos ao longo de 25 anos de investigações. Revelam ainda, o core list dos periódicos que publicam o tema, a estrutura intelectual da pesquisa existente com base em matrizes de cocitações, demonstra como a base conceitual do tema evoluiu, observando que a pesquisa sobre tipo de ameaças tem sido conduzida por sete fluxos teóricos distintos, a saber: (1) temas que investigam os obstáculos que influenciam no crescimento e o no desenvolvimento das pequenas organizações; (2) temas que discutem o estudo do gênero e as dificuldades enfrentadas pelas mulheres no comando das pequenas organizações; (3) temas que investigam as barreiras ao comércio eletrônico das PMEs na percepção dos proprietários/gerentes; (4) temas que abordam as barreiras à implementação dos programas de responsabilidade social empresarial nas pequenas e médias organizações - PMEs; (5) desafios e implicações das escolhas pelo auto emprego; (6) relacionamento entre organizações e credores; e, (7) a performance das pequenas organizações: barreiras, capital humano e financeiro. O presente trabalho está estruturado em 5 seções: a primeira é a introdução, apresentando as motivações e objetivos da presente pesquisa; a segunda seção contempla uma breve revisão da literatura sobre o tema ameaças às pequenas organizações; a terceira seção conta com a metodologia da pesquisa, as explicações referentes ao uso da técnica bibliométrica, amostra e procedimentos de análise; na quarta seção são apresentados os resultados e contempla a análise dos periódicos, análise das citações e análise fatorial; na quinta seção são apresentadas as conclusões, discussões, limitações e futuras avenidas de pesquisa, encerrando com as referências bibliográficas.

\section{Referencial Teórico}

Um ambiente de negócios em constante mudanças é submetido a vários fatores que podem implicar no desempenho das organizações (Watson, 2006; Kwong et al., 2012), ameaçando-as do ponto de vista da sua sobrevivência e do seu crescimento. Estudiosos têm buscado examinar quais os tipos de ameaças (riscos, problemas, dificuldades, obstáculos, barreiras) vêm acometendo as pequenas organizações e até que ponto essas experiências podem contribuir para compreender se uma pequena organizacão possui ou não a capacidade de responder positivamente às interferências ambientais, sociais e econômicas. Acredita-se que conhecer o ambiente dos empreendedores potenciais e reais, os fatores que afetam a auto eficácia e a forma como os indivíduos avaliam as suas capacidades empreendedoras, pode
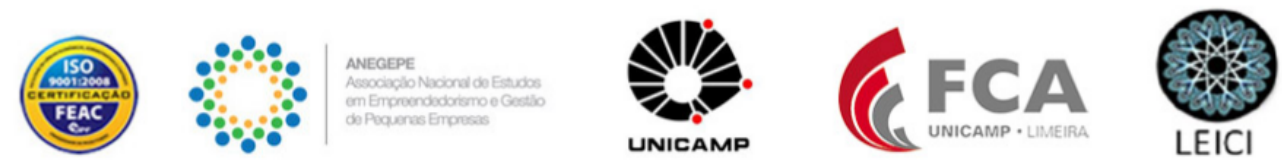
contribuir para o desenvolvimento de um ambiente favorável para criar o sucesso empreendedor (Chen, Greene \& Crick, 1998).

Os estudos envolvendo o tema ameaças estão vinculados especialmente às pesquisas da área de estratégia. Nesta área, algumas lentes teóricas parecem nortear os estudos sobre o tema. A principal delas envolve os estudos que buscam compreender as ameaças enquanto problemas estratégicos e operacionais, associando-os com as etapas do modelo de estágio de crescimento dos negócios (Miles \& Snow, 1978; Porter, 1980; Midgley, 1981; Block \& MacMillan, 1985; Kazanjian, 1988; Orser, Hogarth-Scott \& Riding, 2000; Mambula, 2002; Yasuda, 2005; Robson \& Obeng, 2008). Nessa vertente, as ameaças enfrentadas pelos proprietários das organizações correlacionam-se a uma determinada etapa de crescimento e o processo de resolução dos problemas é repetido em ciclos de crescimento subsequentes (Kazanjian, 1988). Para Cowan (1988) citado por Moy e Luk (2003) a identificação e resolução destas ameaças pode levar a organização para a fase do crescimento.

São muitas as ameaças às pequenas organizações e Kzanjian (1988) sugere que: os problemas relacionados à fase de concepção e desenvolvimento abarcam a fonte e apoio financeiro/financiamento das organizações; os problemas relacionados à fase de comercialização de produtos, compreendem as competências técnicas; os problemas relacionados à fase de crescimento, abarcam a produção, venda e distribuição dos produtos e a pressão para alcançar a rentabilidade desejada; e os problemas relacionados à fase de estabilidade da empresa, envolvem a manutenção do impulso do crescimento e da posição do empreendimento no mercado. Estas ameaças condicionam o sucesso ou o fracasso dos empreendedores e estão vinculados aos problemas internos da empresa.

Um outro olhar dos estudiosos sobre as ameaças que levam ao sucesso ou ao fracasso das pequenas organizações, envolve os problemas originários do padrão de crescimento das organizações maduras, mudança de domínio, engenharia e a administração (Miles \& Snow, 1978), e as dificuldades relativas às tarefas funcionais e ao posicionamento estratégico das organizações (Porter, 1980; Midgley,1981; Moore \& Tushman, 1982; Kazanjian, 1988). Estes problemas são subdivididos de acordo com o constructo organizacional-administrativo e suscitam as preocupações relacionadas: aos sistemas de informação, gerenciamento, controle de custos, sistemas de informação financeira e o nível geral de burocracia; às pessoas, contemplando os problemas relacionados à gestão de pessoas e talento; às vendas e marketing, envolvendo os problemas relacionados às vendas, lucro, mercado, qualidade do produto e suporte ao cliente; problemas relacionados à produção, contemplando a capacidade de produção e desenvolvimento de uma rede de fornecedores confiáveis; e ao posicionamento estratégico, que tratam dos problemas relacionados à posição de uma empresa em um novo segmento de mercado e o desenvolvimento de um novo produto (Kazanjian, 1988).

Nichter e Goldmark (2009) destacaram quatro tipos de fatores que ameaçam o crescimento e o desempenho das organizações comerciais: as características empresariais individuais, as características firmes; os fatores relacionais, como redes sociais ou cadeias de valor; e os fatores contextuais como os obstáculos econômicos e comerciais. Os arranjos institucionais formais sob a forma de legislações, políticas e programas governamentais, também desempenham um papel importante na facilitação do crescimento e desenvolvimento das organizações (Aidis, 2005; Roxas et al., 2013).
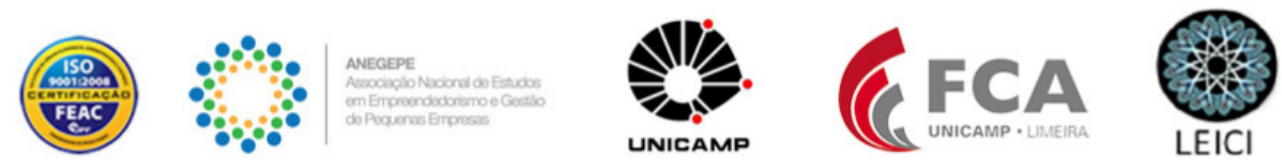
Orser, Hogarth-Scott e Riding (2000) sugeriram como ameaças às organizações, problemas gerenciais. De acordo com os autores, esses problemas podem variar de acordo com os atributos da empresa e com problemas relacionados à falta de conhecimento sobre fontes alternativas de financiamento e captação de recursos. Já Robson e Obeng (2008) mencionaram que as duas maiores ameaças enfrentadas pelas pequenas organizações são a alta taxa da inflação e as altas taxas de juros. Os autores sugeriram que as organizações que empregam membros da família estão mais propensas a enfrentar problemas financeiros do que as organizações não familiares; que organizações localizadas em aglomerações estão mais propensas a enfrentar barreiras de infraestrutura; que o tamanho dos negócios e do setor está fortemente relacionado com a probabilidade de as organizações se depararem com ameaças; que as organizações em crescimento são mais propensas do que outras a enfrentarem ameaças; que o fato de ser ou não exportadora não está correlacionado com problemas de negócios; que as organizações que inovam estão mais propensas a enfrentarem problemas gerenciais e técnicos; as organizações não inovadoras enfrentam ameaças como o alto custo das tarifas da utilidade e a baixa qualidade da eletricidade e do abastecimento de água (Robson \& Obeng, 2008). Com relação às características dos empreendedores, os autores sugeriram que o sexo e a idade não estão relacionados às barreiras nos negócios e que essas descobertas permitem que o governo analise os fatores que podem ser melhorados, especialmente aqueles relacionados à infraestrutura, corrupção, licenciamento e burocracia e altas taxas de juros mencionados como ameaças às pequenas organizações localizadas em aglomerações (Robson \& Obeng, 2008).

As ameaças também foram investigadas nas pesquisas de gênero. Ali e Shabir (2017) constataram que os homens percebem como obstáculos comerciais o transporte, as práticas dos concorrentes, o acesso à terra, os crimes, roubo e desordem, o acesso ao financiamento, a licença comercial, os tribunais, e a regulamentação trabalhista; já as mulheres mencionaram como obstáculos, as altas taxas dos impostos e a corrupção. De acordo com os autores, "os resultados da pesquisa pressupõem que não há diferença nos obstáculos comerciais entre as organizações masculinas e femininas; porém, as organizações de propriedade masculina e feminina variam significativamente em 10 obstáculos no total de 16 parâmetros". (Ali \& Shabir, 2017, p. 10).

Apesar do crescente reconhecimento da importância da identificação das fontes das ameaças por parte dos tomadores de decisões das pequenas organizações, e da relação entre a capacidade de resposta a essas ameaças e a sua sobrevivência, ainda assim, poucas pesquisas examinaram e avaliaram o estoque de conhecimento gerado pela comunidade científica sobre os tipos de ameaças por elas enfrentados. O uso da bibliometria permite fazer algumas deduções a respeito do comportamento dos pesquisadores sobre o tema ameaças às pequenas organizações, bem como identificar algumas tendências de pesquisa em torno dos temas.

\section{Metodologia}

Este artigo é resultado de um estudo bibliométrico cujo propósito é o de apresentar índices de produção e de disseminação do conhecimento científico em uma determinada área (Araujo, 2006). Trata-se de um conjunto de métodos de pesquisa que se vale de análises quantitativa, estatística e de visualização de dados que mapeiam a estrutura do conhecimento em determinado campo científico. Os estudos bibliométricos permitem, dentre outras, a realização de análises do comportamento dos pesquisadores na construção do conhecimento
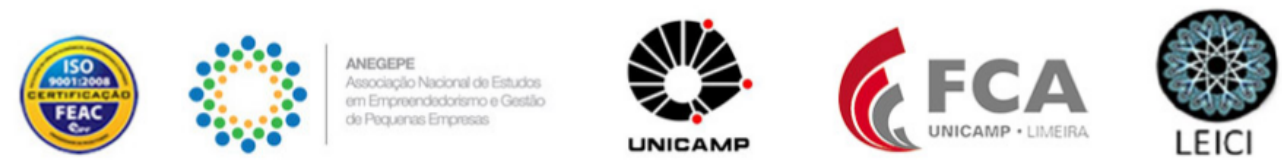
(Vanti, 2002), a identificação de temas que já foram tratados sobre determinado assunto, seus respectivos campos do conhecimento, as inconsistências teóricas nos estudos já tratados e as lacunas ainda não exploradas (Cooper e Lindsay, 1998). Esse estudo focaliza e utiliza as metodologias de análises de citações e cocitações. As bases de dados Scopus e a Web of Science - WoS foram usadas como ferramenta para a seleção de dados por facilitar a pesquisa bibliométrica. As chaves de busca foram os termos *Types of thereats* or *obstacles * or *barriers* or *dificulties*, and *small business* nos títulos, resumos e/ou palavras-chave, restringindo-a somente aos artigos científicos refinados na área de Management, Business e Women Studies, totalizando 953 artigos revisados por especialistas, no período de 1993 a 2017, com 21.467 citações. O recorte para a análise fatorial exploratória foi de artigos com até 4 citações.

3.1 Amostra da Pesquisa: A Figura 1 apresenta a evolução das publicações e número de citações sobre tipos de ameaças aos pequenos negócios no período de 1993 a 2017. Nota-se o crescimento exponencial das publicações sobre o tema durante todo o período da amostra selecionada.

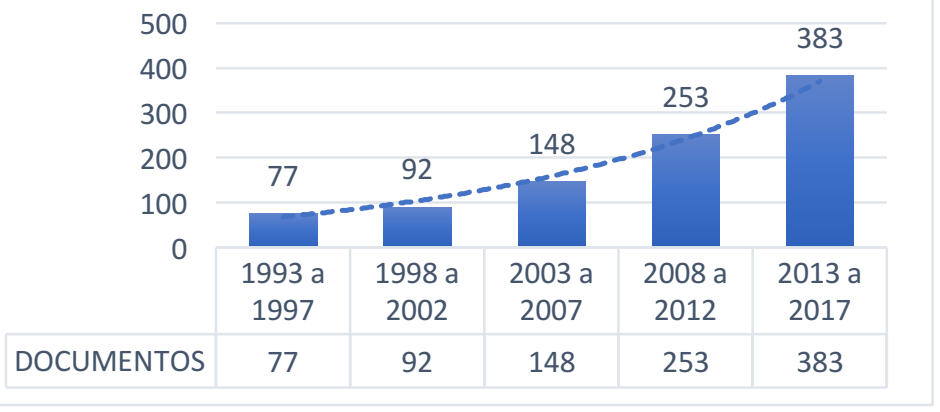

Figura 1 - Evolução das publicações sobre tipos de ameaças às pequenas organizações

Fonte: Elaborado pelos autores base de dados WoS, 2017

3.2. Procedimentos de Análise: $\mathrm{O}$ estudo inclui análise dos periódicos; análise de citações tanto para todo o período quanto em períodos de 5 anos para permitir uma análise da perspectiva longitudinal do fenômeno em questão; análises de cocitações e análise fatorial. Primeiro, identificamos o core list dentre todos os periódicos (ver Figura 2). No segundo procedimento foi realizada a análise das citações coletando todas as referências dos 953 artigos da amostra, ajustando, classificando e resumindo os dados. Relatamos os cinquenta artigos mais influentes ou altamente citados, conforme determinado pelas contagens de citações (ver Figura 3). No terceiro procedimento realizamos a análise fatorial, usando as matrizes de cocitações, com rotação varimax. As cargas fatoriais indicaram o quão bem o artigo pertence ao fator.

\section{Resultados}

4.1 Análise dos periódicos: Para identificar os periódicos com maior produtividade (core list), aplicamos a Lei de Bradford que dispõe que o total de artigos deve ser dividido por três e o grupo de periódicos que tiver mais artigos, até o total de $1 / 3$ dos artigos publicados, é o core do assunto em questão. Na Figura 2 é possível observar que os artigos pertencentes à amostra, encontram-se pulverizados em 151 periódicos diferentes. Dentre estes periódicos, 3 concentram o chamado 'core list' sobre o tópico "tipos de ameaças", contemplando 352 artigos - 36,9\% do total de artigos publicados. 


\begin{tabular}{|l|c|l|c|c|}
\hline \multicolumn{1}{|c|}{ Ranking de Periódicos } & Total de artigos & $\mathbf{\%}$ & Fator de Impacto & Área \\
\hline 1. International Small Business Journal & 119 & 12,5 & 3,677 & Busines \& Management \\
\hline 2. Journal Small Business and Enterprise Development & 119 & 12,5 & 1,98 & Busines \& Management \\
\hline 3. Journal of Business Venturing & 114 & 11,9 & 5.774 & Business \& Management \\
\hline
\end{tabular}

Figura 2 Core da amostra dos periódicos

Fonte: Elaborado pelos autores - base de dados Scopus (2017)

4.2 Análise das citações: Uma importante etapa da bibliometria é a análise de citações, por permitir a identificação e descrição de uma série de padrões na produção do conhecimento científico (Araujo, 2006), "além de fornecer aos leitores, referências importantes sobre o campo de estudo em questão e a contribuição dos autores predecessores para o trabalho atual" (Caldas \& Tinoco, 2004, p. 101). A Figura 3 exibe os artigos mais citados, tanto durante todo o período 1993 a 2017 quanto para cada período de 5 anos. As colunas revelam a frequência absoluta e uma medida relativa da frequência com que cada trabalho é citado no período. Os artigos mais citados e, portanto, mais influentes sobre tipos de ameaças, foram publicados entre 1994 e 2006. Os artigos de Grant (1996) e Amabile et al. (1996) foram os artigos mais citado em quatro períodos consecutivos - 1998 a 2002, 2003 a 2007; 2008 a 2012 e 2013 a 2017, mantendo a sua influência e poder de impacto nas pesquisas sobre tipos de ameaças às pequenas organizações. As células sombreadas destacam as obras que mais se destacaram em cada um dos períodos analisados. O artigo de Grant (1996), por exemplo, retrata as condições instáveis causadas pela inovação e o aumento da intensidade e da diversidade da concorrência que juntos, resultam em capacidades organizacionais, tornando-se a base principal sobre a qual as organizações estabelecem suas estratégias de longo prazo; analisa os mecanismos através dos quais o conhecimento é integrado nas organizações para criar capacidade e estabelecer a vantagem competitiva; menciona as dificuldades na criação das capacidades dinâmicas e de resposta flexível como sendo críticas para o sucesso em mercados hipercompetitivos. Pelo ângulo de análise do autor, a concorrência dos mercados é o tipo de ameaça que merece atenção por parte dos empreendedores. No artigo de Amabile et al. (1996), os autores avaliam o clima para a criatividade, os estímulos percebidos e os obstáculos à criatividade no ambiente organizacional. Sørensen e Stuart (2000) investigam a relação entre envelhecimento organizacional e processos de inovação para iluminar a dinâmica das indústrias de alta tecnologia e resolver os debates na teoria organizacional sobre os efeitos do envelhecimento no funcionamento organizacional e as suas consequências na inovação organizacional. Bechky (2003) mencionou a importância do compartilhamento do conhecimento nas organizações e sugeriu que as dificuldades de conhecimento das comunidades e as diferenças na linguagem entre os membros da organização ao conceituar um produto, são consideradas ameaças à organização. Ghemawat (2001) estudou as dificuldades de criar novos mercados estrangeiros e explorou como as barreiras criadas pelas distâncias cultural, administrativa ou política, geográfica e econômica. Para o autor, essas barreiras podem afetar os diferentes tipos de indústrias, ao tempo em que mostra o quão dramaticamente uma consideração explícita da distância pode mudar a imagem de uma empresa e das suas opções estratégias. Barley (1996) evidenciou as dificuldades que surgem quando as organizações optam por empregar funcionários com perfis técnico e não conseguem apreciar as suas competências e a natureza do seu trabalho. Schoemaker (1993) examinou como o planejamento de cenários contribui no enfrentamento dos desafios metodológicos, organizacionais e psicológicos enfrentados pelos gerentes seniores; os benefícios e os
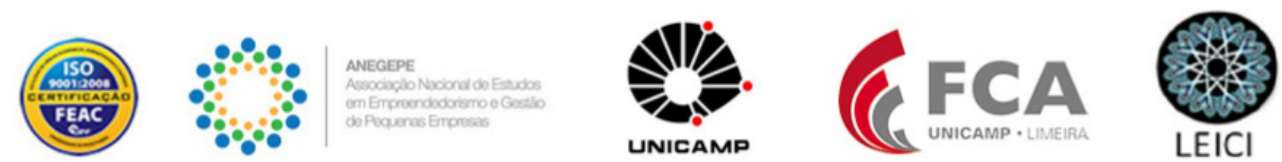
obstáculos à utilização de cenários nas organizações são identificados. Doughert e Heller (1994) construíram uma teoria com o propósito de entender as persistentes dificuldades com a inovação de produtos bem-sucedidas em grandes organizações estabelecidas; concluíram ao sugerirem algumas novidades sobre o por quê das barreiras à inovação existem em grandes organizações estabelecidas e como essas barreiras podem ser gerenciadas. Ohlott, Ruderman e McCauley (1994) analisaram as razões pelas quais poucas mulheres foram promovidas para cargos de alto nível; os resultados sugeriram que os homens experimentam desafios no desenvolvimento das tarefas e as mulheres, experimentam maiores desafios na sua carreira.

Ao analisar os estudos acima mencionados, é possível inferir que as investigações sobre as ameaças evoluiu com o passar dos anos, passando da preocupação com os desafios metodológicos, organizacionais e psicológicos enfrentados pelos gestores seniores (Schoemaker, 1993); aos desafios enfrentados pelas organizações que inovam (Doughert \& Heller, 1994; Sørensen \& Stuart, 2000); às questões do gênero mercado de trabalho (Ohlott, Ruderman \& McCauley, 1994); às dificuldades na criação das capacidades dinâmicas e de resposta flexível, características dos mercados hipercompetitivos (Grant, 1996); às barreiras à criatividade (Amabile, 1996); às dificuldades da gestão de pessoas e aproveitamento da capacidade técnica (Barley, 1996); ao distanciamento cultural na criação de novos mercados (Ghemawat, 2001); e ao compartilhamento do conhecimento e comunicação (Bechky, 2003). Com exceção da inovação, que foi tema de dois dentre os oito artigos mais citados por período, os demais temas demonstraram motivações diferentes por parte dos estudiosos, embora todos eles tenham focado em aspectos ameaçadores às organizações.

A análise de citações também permite identificar quais as obras perceberam uma diminuição relativa nas citações, com destaque para as obras de Barley (1996), Doughert \& Heller (1994) e Ohlott, Ruderman, \& McCauley (1994) que, com o passar dos anos, deixaram de ser interessantes aos olhos dos estudiosos sobre o tema ameaças às pequenas organizações; e as obras que experimentam o aumento relativo citações devido às suas contribuições teóricas, práticas e sociais, como as obras de Sørensen \& Stuart (2000), Ghemawat (2001), Bechky (2003), Ghemawat (2001). Esses resultados podem evidenciar uma menor ênfase no interesse pelas investigações sobre as dificuldades relacionadas à gestão de pessoas; gerenciamento de barreiras à inovação; gênero e ocupações; e o aumento do interesse pelos efeitos do envelhecimento no funcionamento organizacional; obstáculos na criação de mercados estrangeiros; diferenças de linguagens e o compartilhamento das informações. Esses resultados também demonstram mudança conceitual do significado de ameaças à sobrevivência e crescimento das pequenas organizações. 


\begin{tabular}{|c|c|c|c|c|c|c|c|c|c|c|c|c|}
\hline \multirow[t]{3}{*}{ Documentos } & \multirow{2}{*}{\multicolumn{2}{|c|}{$\begin{array}{c}1993-1997 \\
(n=79)\end{array}$}} & \multirow{2}{*}{\multicolumn{2}{|c|}{$\begin{array}{c}1998-2002 \\
(n=469)\end{array}$}} & \multirow{2}{*}{\multicolumn{2}{|c|}{$\begin{array}{r}2003-2007 \\
(n=1526)\end{array}$}} & \multirow{2}{*}{\multicolumn{2}{|c|}{$\begin{array}{r}2008-2012 \\
(n=4042)\end{array}$}} & \multirow{2}{*}{\multicolumn{2}{|c|}{$\begin{array}{r}2013-2017 \\
(n=5146)\end{array}$}} & \multirow{2}{*}{\multicolumn{2}{|c|}{$\begin{array}{l}1993-2017 \\
(n=11.262)\end{array}$}} \\
\hline & & & & & & & & & & & & \\
\hline & $n$ & $\%$ & $n$ & $\%$ & $n$ & $\%$ & $n$ & $\%$ & $n$ & $\%$ & $n$ & $\%$ \\
\hline Grant (1996) & 16 & 20,3 & 136 & 29 & 406 & 26,6 & 681 & 16,8 & 655 & 12,7 & 1894 & 16,8 \\
\hline Amabile etal. (1996) & 3 & 3,8 & 66 & 14,1 & 158 & 10,4 & 543 & 13,4 & 669 & 13 & 1439 & 12,8 \\
\hline Sørensen \& Stuart (2000) & 0 & 0 & 15 & 3,2 & 94 & 6,2 & 184 & 4,6 & 256 & 5 & 549 & 4,9 \\
\hline Bechky (2003) & 0 & 0 & 0 & 0 & 58 & 3,8 & 194 & 4,8 & 266 & 5,2 & 518 & 4,6 \\
\hline Ghemawat (2001) & 0 & 0 & 0 & 0 & 36 & 2,4 & 193 & 4,8 & 271 & 5,3 & 500 & 4,4 \\
\hline Cabrera \& Cabrera (2002) & 0 & 0 & 0 & 0 & 30 & 2 & 167 & 4,1 & 158 & 3,1 & 355 & 3,2 \\
\hline Barley (1996) & 3 & 3,8 & 48 & 10,2 & 72 & 4,7 & 81 & 2 & 64 & 1,2 & 268 & 2,4 \\
\hline March \& Sutton (1997) & 0 & 0 & 13 & 2,8 & 46 & 3 & 94 & 2,3 & 95 & 1,8 & 248 & 2,2 \\
\hline Park \& Ungson (2001) & 0 & 0 & 1 & 0,2 & 21 & 1,4 & 91 & 2,3 & 121 & 2,4 & 234 & 2,1 \\
\hline Schoemaker (1993) & 8 & 10,1 & 13 & 2,8 & 35 & 2,3 & 80 & 2 & 95 & 1,8 & 231 & 2,1 \\
\hline Serensen (2002) & 0 & 0 & 4 & 0,9 & 31 & 2 & 93 & 2,3 & 74 & 1,4 & 202 & 1,8 \\
\hline Kaplan \& Anderson (2004) & 0 & 0 & 0 & 0 & 8 & 0,5 & 50 & 1,2 & 134 & 2,6 & 192 & 1,7 \\
\hline Das \& Teng (2002) & 0 & 0 & 1 & 0,2 & 22 & 1,4 & 78 & 1,9 & 91 & 1,8 & 192 & 1,7 \\
\hline Voss, Sirdeshmukh \& Voss (2008) & 0 & 0 & 0 & 0 & 0 & 0 & 51 & 1,3 & 118 & 2,3 & 169 & 1,5 \\
\hline Doughert \& Heller (1994) & 10 & 12,7 & 21 & 4,5 & 38 & 2,5 & 58 & 1,4 & 39 & 0,8 & 166 & 1,5 \\
\hline Amabile \& Conti (1999) & 0 & 0 & 8 & 1,7 & 27 & 1,8 & 55 & 1,4 & 70 & 1,4 & 160 & 1,4 \\
\hline Lichtenthaler (2011) & 0 & 0 & 0 & 0 & 0 & 0 & 21 & 0,5 & 138 & 2,7 & 159 & 1,4 \\
\hline Whetten (2009) & 0 & 0 & 0 & 0 & 0 & 0 & 58 & 1,4 & 92 & 1,8 & 150 & 1,3 \\
\hline Roth et al. (2008) & 0 & 0 & 0 & 0 & 0 & 0 & 52 & 1,3 & 92 & 1,8 & 144 & 1,3 \\
\hline Kock (2004) & 0 & 0 & 0 & 0 & 15 & 1 & 65 & 1,6 & 60 & 1,2 & 140 & 1,2 \\
\hline Leonidou (2004) & 0 & 0 & 0 & 0 & 8 & 0,5 & 50 & 1,2 & 80 & 1,6 & 138 & 1,2 \\
\hline Ohlott, Ruderman, \& McCauley (1994) & 19 & 24,1 & 36 & 7,7 & 19 & 1,2 & 36 & 0,9 & 27 & 0,5 & 137 & 1,2 \\
\hline Levinthal \& Warglien (1999) & 0 & 0 & 3 & 0,6 & 42 & 2,8 & 45 & 1,1 & 46 & 0,9 & 136 & 1,2 \\
\hline Kotabe \& Murray (2004) & 0 & 0 & 0 & 0 & 7 & 0,5 & 74 & 1,8 & 54 & 1 & 135 & 1,2 \\
\hline Kotter (1995) & 0 & 0 & 0 & 0 & 2 & 0,1 & 51 & 1,3 & 81 & 1,6 & 134 & 1,2 \\
\hline Kirkman et al. (2002) & 0 & 0 & 0 & 0 & 26 & 1,7 & 57 & 1,4 & 46 & 0,9 & 129 & 1,1 \\
\hline Vaara (2003) & 0 & 0 & 0 & 0 & 20 & 1,3 & 53 & 1,3 & 51 & 1 & 124 & 1,1 \\
\hline Shimizu \& Hitt (2004) & 0 & 0 & 0 & 0 & 11 & 0,7 & 46 & 1,1 & 66 & 1,3 & 123 & 1,1 \\
\hline Greenwood, Hinings \& Brown (1994) & 4 & 5,1 & 13 & 2,8 & 19 & 1,2 & 52 & 1,3 & 32 & 0,6 & 120 & 1,1 \\
\hline Schefczyk (1993) & 5 & 6,3 & 20 & 4,3 & 18 & 1,2 & 31 & 0,8 & 46 & 0,9 & 120 & 1,1 \\
\hline Madhok (1996) & 0 & 0 & 19 & 4,1 & 27 & 1,8 & 42 & 1 & 31 & 0,6 & 119 & 1,1 \\
\hline Dean, Brown \& Bamford (1998) & 0 & 0 & 5 & 1,1 & 24 & 1,6 & 46 & 1,1 & 43 & 0,8 & 118 & 1 \\
\hline Cohen (2007) & 0 & 0 & 0 & 0 & 1 & 0,1 & 43 & 1,1 & 70 & 1,4 & 114 & 1 \\
\hline Olie (1994) & 2 & 2,5 & 6 & 1,3 & 29 & 1,9 & 43 & 1,1 & 33 & 0,6 & 113 & 1 \\
\hline Teng, Cheon \& Grover (1995) & 3 & 3,8 & 1 & 0,2 & 36 & 2,4 & 57 & 1,4 & 14 & 0,3 & 111 & 1 \\
\hline Frizelle \& Woodcock (1995) & 0 & 0 & 10 & 2,1 & 16 & 1 & 41 & 1 & 40 & 0,8 & 107 & 1 \\
\hline Michaelidou, Siamagka \& & 0 & 0 & 0 & 0 & 0 & 0 & 1 & 0 & 105 & 2 & 106 & 0,9 \\
\hline Morris et al. (2006) & 0 & 0 & 0 & 0 & 1 & 0,1 & 48 & 1,2 & 57 & 1,1 & 106 & 0,9 \\
\hline Grégoire, Barr \& Shepherd (2010) & 0 & 0 & 0 & 0 & 0 & 0 & 24 & 0,6 & 81 & 1,6 & 105 & 0,9 \\
\hline Berg, Grant \& Johnson (2010) & 0 & 0 & 0 & 0 & 0 & 0 & 19 & 0,5 & 83 & 1,6 & 102 & 0,9 \\
\hline Kwak, Chih \& Ibbs (2009) & 0 & 0 & 0 & 0 & 0 & 0 & 17 & 0,4 & 83 & 1,6 & 100 & 0,9 \\
\hline Salvador, De Holan \& Piller (2009) & 0 & 0 & 0 & 0 & 0 & 0 & 12 & 0,3 & 88 & 1,7 & 100 & 0,9 \\
\hline Hewlett (2008) & 0 & 0 & 0 & 0 & 15 & 1 & 39 & 1 & 44 & 0,9 & 98 & 0,9 \\
\hline Hill \& Hellriegel (1994) & 6 & 7,6 & 11 & 2,3 & 24 & 1,6 & 31 & 0,8 & 26 & 0,5 & 98 & 0,9 \\
\hline Miettinen \& Virkkunen (2005) & 0 & 0 & - & 0 & 4 & 0,3 & 47 & 1,2 & 45 & 0,9 & 96 & 0,9 \\
\hline Markides (1998) & 0 & 0 & 15 & 3,2 & 29 & 1,9 & 30 & 0,7 & 20 & 0,4 & 94 & 0,8 \\
\hline Madrid -Gujjarro, Garcia \& Van Auken & 0 & 0 & 0 & 0 & 0 & 0 & 23 & 0,6 & 70 & 1,4 & 93 & 0,8 \\
\hline Karagozoglu \& Lindell (1998) & 0 & 0 & 4 & 0,9 & 23 & 1,5 & 35 & 0,9 & 31 & 0,6 & 93 & 0,8 \\
\hline Repenning \& Sterman (2001) & 0 & 0 & 0 & 0 & 28 & 1,8 & 31 & 0,8 & 33 & 0,6 & 92 & 0,8 \\
\hline Eagly \& Carli (2007) & 0 & 0 & 0 & 0 & 0 & 0 & 29 & 0,7 & 62 & 1,2 & 91 & 0,8 \\
\hline
\end{tabular}

Figura 3 Frequência de citação relativa e bruta por período em relação

Fonte: Elaborado pelos autores - base de dados WoS (2017)

Nota: $n$. (total de documentos publicados no período).

4.3 Análise fatorial: A análise fatorial é o método que busca uma solução teórica não contaminada por variabilidade de erro (Tabachnick \& Fidel, 1983). Seu propósito é detectar a estrutura dos dados ou uma modelagem causal por meio de correlações entre as variáveis. Tratase de um método comumente usado nas pesquisas na área de Ciências Sociais (Hair et al., 2005). Na presente pesquisa, as análises evidenciaram que a estrutura da produção científica sobre tipos de ameaças às pequenas organizações é explicada por 32 obras, dentre artigos e livros, com uma variância acumulada de 73,6\%, com Medida Kaiser-Meyer-Olkin - KMO, de adequação de amostragem de 0,89, ambas atendendo ao patamar sugerido. Estas obras foram agrupadas em sete fatores/dimensões (Figura 4). Os fatores que têm um impacto maior são responsáveis por um maior número de trabalhos (Ferreira, Storopoli \& Serra, 2014). 
O primeiro fator abrange o período entre 1994 e 2008 e é composto por 6 artigos e um livro que juntos, explicam $20,4 \%$ da variância. O agrupamento destes artigos remete às questões-chaves envolvendo os problemas, obstáculos, barreiras e as dificuldades enfrentadas pelos proprietários de organizações que influenciam o crescimento dos negócios nos diferentes estágios do seu desenvolvimento. Especificamente, é possível observar a busca dos estudiosos por compreender os padrões existentes nas várias fases do crescimento dos empreendimentos baseados em tecnologia (Kazanjian, 1988); a formação, desenvolvimento, contribuição e a gestão das organizações de pequeno porte, sua relação como sucesso e fracasso (Storey, 1994); problemas/obstáculos enfrentados pelos proprietários/gerentes nas diferentes fases do desenvolvimento comercial (Orser, Hogarth-Scott \& Riding, 2000); o quanto esses fatores implicam na economia (Mambula, 2002); as estratégias usadas por alguns proprietários para superar os problemas/obstáculos (Moy \& Luk, 2003); uma análise do relacionamento entre a taxa de crescimento, tamanho e idade da empresa no setor de produção, o quanto estas variáveis influenciam as atividades de Pesquisa e Desenvolvimento - P\&D, a relação de subcontratação com o crescimento da empresa e a sobrevivência dos negócios (Yasuda, 2005); identifica e busca compreender a natureza do empreendedorismo em um país em desenvolvimento e os problemas/obstáculos enfrentados pelos proprietários/gerentes (Robson \& Obeng, 2008). Com base nas características comuns aos artigos e livro, o Fator 1 foi nomeado "Obstáculos que influenciam no crescimento e o no desenvolvimento das pequenas organizações".

O segundo fator compreende o período de 1996 a 2006 e é constituído por 5 artigos e um livro que juntos, explicam $14,82 \%$ da variância. A integração destas obras remete às discussões acerca do empoderamento das mulheres empreendedoras, das discussões sobre o gênero e do interesse da mulher pela propriedade da empresa (Carter, Anderson \& Shaw, 2000); versa sobre as políticas assistenciais e as especificidades no estudo do gênero (Matthews \& Moser, 1996); analisa comparativamente o desempenho e o crescimento das organizações comandas por mulheres brancas e as pertencentes ao grupo 'minoritário' (Ram \& Smallbone, 2003); aborda as questões relativas às experiências e barreiras enfrentadas pelas mulheres asiáticas (Dawe \& Fielden (2005) e a importância das políticas públicas e das organizações que incentivam o desenvolvimento e a busca de novas oportunidades no auxílio às mulheres; busca explicar como o capital social e os fatores pessoais (vieses cognitivos) interagem e influenciam o comportamento empreendedor na exploração de oportunidades (De Carolis \& Saparito, 2006). Esses artigos sugerem a existência de um foco na motivação das mulheres para empreender. Sugerem algumas dificuldades por elas enfrentadas relativas ao gênero, e as suas implicações no aproveitamento de novas oportunidades, no comportamento das empreendedoras e na sobrevivência dos negócios. Com base nas características comuns aos artigos e livro, o Fator 2 foi nomeado "Estudo do gênero e as dificuldades enfrentadas pelas mulheres no comando das pequenas organizações".

O terceiro fator compreende o período de 1996 a 2006, sendo integrado por 5 artigos e um livro que juntos, explicam $11,25 \%$ da variância. A integração destas obras reporta-se à necessidade de adaptação e inovação das pequenas organizações para atender ao mercado globalizado e competitivo; à produtividade como dependente da forma como as organizações competem (Porter, 1998; Hadjimonolis, 1999); a expansão dos negócios eletrônicos decorrente da inovação e a percepção dos proprietários/gerentes a respeito das barreiras/obstáculos que podem dificultar essa expansão destes negócios (Porter, 1998; Hadjimonolis 1999; Matlay \&
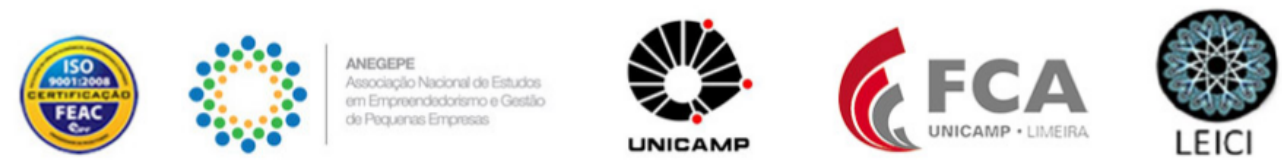
Addis, 2003; Tetteh \& Burn, 2001; Huang \& Brown, 1999; Lawsow et al. 2003). Com base nas características comuns aos artigos e livro, o Fator 3 foi nomeado "Barreiras ao comércio eletrônico das pequenas e médias organizações na percepção dos proprietários/gerentes".

O quarto fator compreende o período de 1991 a 2006, sendo integrado por três artigos e um livro que juntos, explicam $9,22 \%$ da variância. A integração destes artigos remete à relação das pequenas organizações com a sociedade (Goss, 1991); ao desenvolvimento da teoria da responsabilidade social das pequenas organizações (Lepoutre \& Heene, 2006); a reflexão acerca da terminologia da RSE nas PMEs analisada a partir das ações sociais e práticas ambientais adotadas (Murillo \& Lozano, 2006); a distinção entre as várias abordagens teóricas que foram aplicadas sobre a RSE nas grandes corporações e PMEs (Perrini, 2006). Dentre os vários contextos abordados, os autores debatem sobre as dificuldades enfrentadas pelas PMEs na adoção das práticas socialmente responsáveis; das dificuldades de conscientizar os funcionários; das barreiras práticas para se envolver em atividades de SBSR; as dificuldades de reconhecimento das características da RS e das capacidades cognitivas limitadas; os problemas relacionados à terminologia das ações socialmente responsáveis, dentre outras. Com base nas características comuns aos artigos e livro, o Fator 4 foi nomeado "Responsabilidade Social Empresarial nas pequenas e médias organizações: barreiras na concepção e prática".

O quinto fator compreende o período de 1982 a 2000 e incorpora três artigos e um livro que juntos, explicam $7,47 \%$ da variância. Reflete acerca das características do empreendedor, da sua capacidade de criar mercados, fornecedores e clientes e o quanto esses elementos implicam no crescimento das organizações, na mobilidade social e na cultura empresarial (Casson, 1982); exploram os fatores que levam os indivíduos ao trabalho por conta própria (Evans \& Leighton,1989); ao empreendedorismo no setor agrícola no Reino Unido, analisado à partir das organizações rurais indígenas (Carter \& Rosa, 1998); ao gênero e as práticas de trabalho em pequenas organizações (Baines \& Wheelock, 2000). As características comuns aos artigos e livro, permitem nomear o Fator 5 em "As implicações das escolhas pelo auto emprego".

O sexto fator compreende o período de 1990 a 1998 e incorpora três artigos, explicando $6,32 \%$ da variância. Envolvem discussões sobre o relacionamento entre uma empresa e seus credores e a medida em que essas organizações são nutridas e têm acesso ao capital necessário para o crescimento (Petersen \& Rajan, 1994); o processo de empréstimo e a assimetria de informação que permite aos credores capturar algumas das rendas geradas pelos seus clientes mais antigos (Sharpe, 1990); a relação entre empréstimos bancários a pequenas organizações, tamanho e complexidade da empresa bancária e consolidação bancária (Strahan \& Weston, 1998). As características comuns aos artigos permitem nomear o Fator 6 em "Relacionamento entre organizações e credores".

O sétimo fator é formado por dois artigos, publicados em 1994 e 2004, explicado por uma variância de 4,06\%. Nesses artigos, os autores buscam prever o desempenho de novos empreendimentos com base em fatores do capital humano e financeiro observados no momento do arranque da empresa, sob três resultados possíveis: (1) falha, (2) sobrevivência marginal ou (3) alto crescimento (Cooper, Gimeno-Gascon \& Woo, 1994); os obstáculos do caminho da internacionalização das organizações de pequeno porte e os impactos às barreiras internas e externas à exportação (Leonidou, 2004). As características comuns aos artigos permitem nomear o Fator 7 como "Performance das pequenas organizações: barreiras, capital humano e
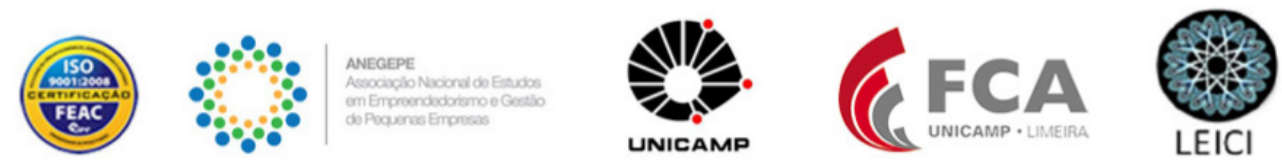
financeiro". Em síntese, a Figura 4 apresenta os sete fatores, com os principais autores por fator, o número de citações no Google Scholar.

\begin{tabular}{|c|c|c|c|c|}
\hline Fator 1 & Autores & Cit. Google & Comunal & $\begin{array}{l}\text { Variância } \\
\text { Explicada }\end{array}$ \\
\hline \multirow{7}{*}{$\begin{array}{l}\text { Obstáculos que influenciam no } \\
\text { crescimento e o no desenvolvimento } \\
\text { das pequenas organizações }\end{array}$} & Robson \& Obeng (2008) & 90 & 0,937 & \multirow{7}{*}{$20,40 \%$} \\
\hline & Moy \& Luk (2003) & 43 & 0,935 & \\
\hline & Yasuda (2005) & 380 & 0,924 & \\
\hline & Mambula (2002) & 183 & 0,878 & \\
\hline & Orser, Hogarth-Scott \& Riding (2000) & 328 & 0,878 & \\
\hline & Kazanjian (1988) & 1.214 & 0,865 & \\
\hline & Storey (1994) & 5.944 & 0,807 & \\
\hline Fator 2 & Autores & Cit. Google & Comunal & $\begin{array}{l}\text { Variância } \\
\text { Explicada }\end{array}$ \\
\hline \multirow{6}{*}{$\begin{array}{l}\text { Estudo do gênero e as dificuldades } \\
\text { enfrentadas pelas mulheres no } \\
\text { comando das pequenas organizações }\end{array}$} & De Carolis \& Saparito (2006) & 684 & 0,972 & \multirow{6}{*}{$14,82 \%$} \\
\hline & Ram \& Smallbone (2003) & 134 & 0,937 & \\
\hline & Dawe \& Fielden (2005) & 20 & 0,898 & \\
\hline & Smith-Hunter \& Boyd (2004) & 110 & 0,898 & \\
\hline & Matthews \& Moser (1996) & 349 & 0,883 & \\
\hline & Carter, Anderson \& Shaw (2000) & 369 & 0,79 & \\
\hline Fator 3 & Autores & Cit. Google & Comunal & $\begin{array}{l}\text { Variância } \\
\text { Explicada }\end{array}$ \\
\hline \multirow{6}{*}{$\begin{array}{l}\text { Barreiras ao comércio eletrônico das } \\
\text { PMEs na percepção dos } \\
\text { proprietários/gerentes }\end{array}$} & Hadjimonolis (1999) & 409 & 0,842 & \multirow{6}{*}{$11,25 \%$} \\
\hline & Porter (1998) & 13.462 & 0,842 & \\
\hline & Matlay \& Addis (2003) & 99 & 0,772 & \\
\hline & Tetteh \& Burn (2001) & 200 & 0,772 & \\
\hline & Huang \& Brown (1999) & 280 & 0,755 & \\
\hline & Lawsow et al. (2003) & 217 & 0,755 & \\
\hline Fator 4 & Autores & Cit. Google & Comunal & $\begin{array}{l}\text { Variância } \\
\text { Explicada }\end{array}$ \\
\hline \multirow{4}{*}{$\begin{array}{l}\text { Responsabilidade social empresarial } \\
\text { nas PMEs: barreiras na concepção e } \\
\text { prática }\end{array}$} & Lepoutre \& Heene (2006) & 652 & 0,916 & \multirow{4}{*}{$9,22 \%$} \\
\hline & Murillo \& Lozano (2006) & 567 & 0,916 & \\
\hline & Perrini (2006) & 421 & 0,916 & \\
\hline & Goss (1991) & 283 & 0,808 & \\
\hline Fator 5 & Autores & Cit. Google & Comunal & $\begin{array}{l}\text { Variância } \\
\text { Explicada }\end{array}$ \\
\hline \multirow{4}{*}{$\begin{array}{l}\text { As implicações das escolhas pelo auto } \\
\text { emprego }\end{array}$} & Baines \&Wheelock (2000) & 84 & 0,784 & \multirow{4}{*}{$7,47 \%$} \\
\hline & Carter \& Rosa (1998) & 71 & 0,784 & \\
\hline & Casson (1982) & 3.723 & 0,784 & \\
\hline & Evans \& Leighton (1986) & 2.858 & 0,784 & \\
\hline Fator 6 & Autores & Cit. Google & Comunal & $\begin{array}{l}\text { Variância } \\
\text { Explicada }\end{array}$ \\
\hline \multirow{3}{*}{$\begin{array}{l}\text { Relacionamento entre organizações e } \\
\text { credores }\end{array}$} & Petersen \& Rajan (1994) & 4.874 & 0,889 & \multirow{3}{*}{$6,32 \%$} \\
\hline & Sharpe (1990) & 2.440 & 0,825 & \\
\hline & Strahan \& Weston (1998) & 541 & 0,825 & \\
\hline Fator 7 & Autores & Cit. Google & Comunal & $\begin{array}{l}\text { Variância } \\
\text { Explicada. }\end{array}$ \\
\hline \multirow{2}{*}{$\begin{array}{l}\text { Performance das pequenas } \\
\text { organizações: barreiras, capital humano } \\
\text { e financeiro }\end{array}$} & Cooper, Gimeno-Gascon \&Woo (1994) & 2.362 & 0,834 & \multirow{2}{*}{$4,06 \%$} \\
\hline & Leonidou (2004) & 629 & 0,828 & \\
\hline
\end{tabular}

Figura 4- Fluxo teórico das pesquisas sobre ameaça à pequenas organizações.

Fonte: Elaborado pelas autoras - base de dados Scopus (2017)

\section{Conclusões}

Neste trabalho, objetivamos examinar e dar sentido às pesquisas sobre as ameaças à pequenas organizações. Os resultados permitiram identificar as obras mais influentes, os principais periódicos, o desenvolvimento das pesquisas sobre tipos de ameaças e os fluxos teóricos sobre as ameaças às pequenas organizações, propiciando avançar os laços intelectuais 
e a evolução das pesquisas sobre esse tema no campo do empreendedorismo. Os achados da pesquisa complementam os estudos realizados sobre os tipos de ameaças à medida em que identifica sete fluxos teóricos distintos sobre o tema "ameaças às pequenas organizações". Além disso, reforça o interesse e a motivação dos pesquisadores em investigar as ameaças ambientais com temas que retratam a concorrência, e ressalta as dificuldades enfrentadas pelas mulheres na identificação de novas oportunidades, investimento e desenvolvimento dos seus negócios. Sugere que as dificuldades no âmbito da gestão administrativa, gestão de pessoas e talentos humanos, vendas e marketing e posicionamento estratégico refletem uma grande preocupação por parte dos empreendedores. Esses achados foram consistentes com o estudo de Nichter e Goldmaark (2009) que destacou quatro tipos de fatores que afetam o crescimento e o desempenho das organizações comerciais: as características empresariais individuais; as características firmes; os fatores relacionais, como redes sociais ou cadeias de valor; e os fatores contextuais como os obstáculos comerciais. Identificamos, também, que os interesses e investigações sobre as ameaças foram modificando com o tempo, e essas mudanças tem uma estreita relação com o contexto estudado e com o mercado. Os estudos que mais influenciaram o tema ameaças, foram os de Schoemaker (1993), Doughert e Heller, (1994), Sørensen e Stuart (2000), Ohlott, Ruderman e McCauley (1994), Grant (1996), Amabile (1996), Barley (1996) e Ghemawat (2001) e Bechky (2003). Por meio da análise bibliométrica, foi possível identificar os sete fluxos teóricos potenciais, elevando o pressuposto central das pesquisas sobre os tipos de ameaças a um amadurecimento e, assim, observamos a ampliação das suas fronteiras para além da área da estratégica, fato esse suficiente para justificar um exame da literatura existente.

Os resultados também sugerem que as investigações mais citadas permanecem influentes nos estudos sobre o tipo de ameaças e que mainstream das investigações está focado na busca por compreender vários desafios, entre eles os de caráter metodológico, organizacional e psicológico enfrentados pelos gestores seniores. Outros desafios enfrentados pelas organizações, voltam-se para questões da inovação, aquelas relativas ao empoderamento feminino no mercado de trabalho, dificuldades na criação das capacidades dinâmicas e de resposta flexível características dos mercados hipercompetitivos. Além dessas, as barreiras relativas à criatividade, dificuldades enfrentadas pela gestão de pessoas e ao distanciamento cultural na criação de novos mercados se fazem presentes. Encontramos ainda, compartilhamento do conhecimento e da comunicação, dificuldades ao optar pelo auto emprego, além das ameaças ao desempenho das pequenas organizações. Muitos desses estudos também relacionaram as ameaças com as etapas do modelo de estágio de crescimento dos negócios. Por fim, acreditamos que esse estudo bibliométrico pode contribuir com o avanço das pesquisas sobre a tipologia das ameaças às pequenas organizações ao fornecer uma análise quantitativa das pesquisas já realizadas sobre o tópico na área de negócios e por criar novas perspectivas para futuras pesquisas no campo do empreendedorismo. Embora tenhamos identificados que as obras clássicas permanecem influentes nas pesquisas sobre tipos de ameaças, existe uma nova corrente de investigações que começam a discutir esse tema em contextos diferentes e que podem contribuir para ampliar os pontos de vista dos especialistas e profissionais especialmente no campo do empreendedorismo. Concluímos a presente pesquisa, acreditando que com novas investigações envolvendo informações adicionais sobre a psicologia cognitiva, por exemplo, é possível que se obtenha o aprimoramento do reconhecimento dos tipos de ameaças e a influência no comportamento de enfrentamento dos
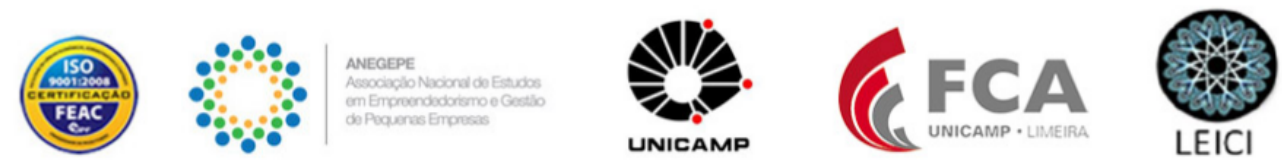
empreendedores ao vivenciarem situações de ameaças. Esse estudo também contribui à media em sinaliza novas avenidas para investigação, capazes de gerar contribuições teóricas (desenvolvimento do campo do empreendedorismo), sociais (redução da mortalidade das pequenas organizações, geração de emprego e renda); e gerenciais (na medida em que um empreender reconhece as suas emoções ao lidar com as ameaças, provavelmente a sua percepção de risco poderá mudar o seu estado de alerta, fazendo com que consiga reagir e superar esses obstáculos). Devemos nos lembrar que o fator humano é um recurso importante e que pode influenciar o desempenho das organizações. As suas emoções, nível de motivação, nível de educação e habilidades são determinantes para o sucesso do negócio.

\subsection{Limitações e futuras avenidas de pesquisa}

Este estudo tem algumas limitações a serem avaliadas. A escolha pelas técnicas bibliométricas aqui empregadas promovem algumas discussões acerca da não análise dos contextos em que os artigos foram desenvolvidos, dos propósitos das citações, conteúdos dos trabalhos, bases teóricas adotadas, dentre outras lacunas que juntas, contribuem para o avanço das pesquisas sobre ameaças às pequenas organizações. A adoção por softwares de análise de conteúdo pode contribuir para uma melhor contextualização do fenômeno estudado (Ferreira, Storopoli \& Serra, 2014). Primeiro, a base teórica poderia ter sido ampliada com uma análise cuidadosa dos artigos mais influentes e a adoção de palavras-chave que melhor significassem à evolução e compreensão da pesquisa sobre ameaças às pequenas organizações. Por exemplo, se identificamos que os estudos sobre as ameaças estão concentrados na área de estratégia, ao fazermos um recorte em outras áreas, como nos estudos do gênero, recursos humanos, inovação, setores específicos, poderíamos melhor compreender as tipologias das ameaças. Segundo, os fluxos teóricos aqui apresentados é um olhar inicial dos tipos de ameaças às pequenas organizações, especialmente porque a tipologia das ameaças pode variar de acordo com os atores investigados, porte da empresa, setor, ambiente, gênero e contexto. Sobre o gênero especificamente, embora não tenha sido foco da atenção na presente pesquisa, a percepção das mulheres e dos homens diferem quanto ao tipo de ameaças. Essa informação encontra o apoio de Ali e Shabir (2017) que ao pesquisarem a diferença no desempenho do negócio e nos obstáculos entre organizações de propriedade de homens e mulheres na Índia, identificaram que os homens percebem como obstáculos comerciais o transporte, práticas dos concorrentes, acesso à terra, crimes, roubo e desordem, acesso ao financiamento, licença comercial, tribunais e regulamentação trabalhista; enquanto que as mulheres percebem as altas taxas dos impostos e a corrupção como ameaças aos seus empreendimentos. Uma terceira área para pesquisa futura diz respeito à identificação das emoções experimentadas pelos empreendedores ao se depararem com as situações ameaçadoras. Acreditamos que os sentimentos, emoções e afetos apoiam o raciocínio; e que as informações identificadas serão usadas posteriormente, podendo influenciar o comportamento empreendedor. Essas informações são apoiadas por Damásio (2009), George, 1991, 2000, Forgas, 1995, 2000; Zajonc, 2000; Adolphs \& Damásio; 2001; Isen, 2002; Baron, 2008). Já dizia O'Malley e Gillette (1984), que as estratégias de pesquisa que se propõem a prever o comportamento do empreendedor, precisam confiar nas informações que tratam a respeito das emoções das pessoas, uma vez que elas estão intimamente relacionadas às reações subsequentes. Essa informação também é corroborada por Richard, van der Pligt \& Vries, (1995) que sugerem que quando se antecipa o efeito imediato das emoções e

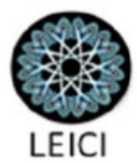


afetividade, torna-se possível prever o comportamento para além dos componentes cognitivos. Quarto, esse estuda também não identificou as ameaças por tempo e experiência do empreendedor. Acredita-se que a experiência faz com que a percepção das ameaças seja diferente. Sendo assim, investigar como os jovens empreendedores percebem as ameaças pode trazer grandes contribuições para o desenvolvimento da tipologia das ameaças às pequenas organizações. Essa investigação pode ser corroborada pelos dizeres de Orser, Hogarth-Scott e Riding (2000), ao investigarem o processo de crescimento das pequenas organizações, os problemas e os obstáculos enfrentados pelo proprietários/gerentes nas diferentes fases do desenvolvimento do negócio, constataram que menos de um quarto das organizações estudadas relataram dois anos consecutivos de aumento de receita; que as organizações em crescimento eram organizações mais jovens, enquanto as organizações em declínio eram comparativamente as mais antigas; e que a presença de um plano de negócios foi altamente correlacionada com o desempenho. Então, até que ponto a percepção das ameaças é igual para os empreendedores jovens e experientes? Como essa percepção pode ter influenciado o comportamento de enfrentamento desses empreendedores?

\section{Referências}

Aidis, R. (2005). Institutional barriers to small-and medium-sized enterprise operations in transition countries. Small business economics, 25(4), 305-317.

Ali, J., \& Shabir, S. (2017). Does gender make a difference in business performance? Evidence from a large enterprise survey data. Gender in Management: An International Journal, 32(3).

Amabile, T. M., Conti, R., Coon, H., Lazenby, J., \& Herron, M. (1996). Assessing the work environment for creativity. Academy of Management Journal, 39(5), 1154-1184.

Amabile, T. M., \& Conti, R. (1999). Changes in the work environment for creativity during downsizing. Academy of Management Journal, 42(6), 630-640.

Araújo, C. A. (2006). Bibliometria: evolução histórica e questões atuais. Em questão, 12(1).

Baines, S., \& Wheelock, J. (2000). Work and employment in small businesses: perpetuating and challenging gender traditions. Gender, Work \& Organization, 7(1), 45-56.

Barley, S. R. (1996). Technicians in the workplace: Ethnographic evidence for bringing work into organizational studies. Administrative Science Quarterly, 404-441.

Bechky, B. A. (2003). Sharing meaning across occupational communities: The transformation of understanding on a production floor. Organization Science, 14(3), 312-330.

Berg, J. M., Grant, A. M., \& Johnson, V. (2010). When callings are calling: Crafting work and leisure in pursuit of unanswered occupational callings. Organization Science, 21(5), 973-994.

Bignetti, L. P., \& Paiva, E. L. (2002). Ora (direis) ouvir estrelas!: estudo das citações de autores de estratégia na produção acadêmica brasileira. Revista de Administração Contemporânea, 6(1), 105-125.

Block, Z., \& MacMillan, I. C. (1985). Milestones for successful venture planning. Harvard Business Review, 63(5), 184.

Cabrera, A., \& Cabrera, E. F. (2002). Knowledge-sharing dilemmas. Organization Studies, 23(5), 687-710.

Carter, S., Anderson, S., \& Shaw, E. (2000). Women's business ownership: A review of the academic, popular and internet literature with a UK policy focus. ARPENT: Annual Review of Progress in Entrepreneurship, 1, 66.

Carter, S., \& Rosa, P. (1998). Indigenous rural firms: farm enterprises in the UK. International Small Business Journal, 16(4), $15-27$.

Casson, M. (1982). The entrepreneur: An economic theory. Rowman \& Littlefield.

Chen, C. C., Greene, P. G., \& Crick, A. (1998). Does entrepreneurial self-efficacy distinguish entrepreneurs from managers?. Journal of business venturing, 13(4), 295-316.

Cohen, M. D. (2007). Reading Dewey: Reflections on the study of routine. Organization Studies, 28(5), 773-786.

Cooper, A. C., Gimeno-Gascon, F. J., \& Woo, C. Y. (1994). Initial human and financial capital as predictors of new venture performance. Journal of Business Venturing, 9(5), 371-395.

De Carolis, D. M., \& Saparito, P. (2006). Social capital, cognition, and entrepreneurial opportunities: A theoretical framework. Entrepreneurship Theory and Practice, 30(1), 41-56.

Evans, D. S., \& Leighton, L. S. (1989). Some empirical aspects of entrepreneurship. The American Economic Review, 79(3), 519-535.

Ghemawat, P. (2001). Distance still matters. Harvard Business Review, 79(8), 137-147.

Greenwood, R., Hinings, C. R., \& Brown, J. (1994). Merging professional service firms. Organization Science, 5(2), $239-257$. 
Hadjimanolis, A. (1999). Barriers to innovation for SMEs in a small less developed country (Cyprus). Technovation, 19(9), 561-570.

Das, T. K., \& Teng, B. S. (2002). Alliance constellations: A social exchange perspective. Academy of Management Review, 27(3), 445-456.

Dawe, A. J., \& Fielden, S. L. (2005). 10 The experiences of Asian women entering business start-up in the UK. International handbook of women and small business entrepreneurship, 120.

Dean, T. J., Brown, R. L., \& Bamford, C. E. (1998). Differences in large and small firm responses to environmental context: Strategic implications from a comparative analysis of business formations. Strategic Management Journal, 709-728.

Dougherty, D., \& Heller, T. (1994). The illegitimacy of successful product innovation in established firms. Organization Science, 5(2), 200-218.

Eagly, A. H., \& Carli, L. L. (2007). Women and the labyrinth of leadership. Harvard Business Review, 85(9), 62.

Goss, D. (1991). Small Business and Society (Routledge Revivals). Routledge.

Ferreira, M. P., Storopoli, J. E., \& Serra, F. R. (2014). Two decades of research on strategic alliances: analysis of citations, cocitations and themes researched. Revista de Administração Contemporânea, 18(SPE), 109-133.

Grant, R. M. (1996). Prospering in dynamically-competitive environments: Organizational capability as knowledge integration. Organization Science, 7(4), 375-387.

Grégoire, D. A., Barr, P. S., \& Shepherd, D. A. (2010). Cognitive processes of opportunity recognition: The role of structural alignment. Organization science, 21(2), 413-431.

Hair, J. F., Black, W. C., Babin, B. J., Anderson, R. E., \& Tatham, R. L. (2006). Multivariate data analysis 6th Edition. Pearson Prentice Hall. New Jersey. humans: Critique and reformulation. Journal of Abnormal Psychology, 87, 49-74.

Hewlett, S. A. (2008). Off-ramps and on-ramps: Keeping talented women on the road to success. Human Resource Management International Digest, 16(2).

Hill, R. C., \& Hellriegel, D. (1994). Critical contingencies in joint venture management: Some lessons from managers. Organization science, 5(4), 594-607.

Huang, X., \& Brown, A. (1999). An analysis and classification of problems in small business. International Small Business Journal, 18(1), 73-85.

Frizelle, G., \& Woodcock, E. (1995). Measuring complexity as an aid to developing operational strategy. International Journal of Operations \& Production Management, 15(5), 26-39.

Kaplan, R. S., \& Anderson, S. R. (2004). Time-driven activity-based costing. Harvard Business Review.

Karagozoglu, N., \& Lindell, M. (1998). Internationalization of small and medium-sized technology-based firms: An exploratory study. Journal of Small Business Management, 36(1), 44.

Kazanjian, R. K. (1988). Relation of dominant problems to stages of growth in technology-based new ventures. Academy of Management Journal, 31(2), 257-279.

Kirkman, B. L., Rosen, B., Gibson, C. B., Tesluk, P. E., \& McPherson, S. O. (2002). Five challenges to virtual team success: Lessons from Sabre, Inc. The Academy of Management Executive, 16(3), 67-79.

Kock, N. (2004). The psychobiological model: Towards a new theory of computer-mediated communication based on Darwinian evolution. Organization Science, 15(3), 327-348.

Kotabe, M., \& Murray, J. Y. (2004). Global sourcing strategy and sustainable competitive advantage. Industrial Marketing Management, 33(1), 7-14.

Kotter, J. P. (1995). Leading change: Why transformation efforts fail.

Kwak, Y. H., Chih, Y., \& Ibbs, C. W. (2009). Towards a comprehensive understanding of public private partnerships for infrastructure development. California Management Review, 51(2), 51-78.

Kwong, C., Jones-Evans, D., \& Thompson, P. (2012). Differences in perceptions of access to finance between potential male and female entrepreneurs: Evidence from the UK. International Journal of Entrepreneurial Behavior \& Research, 18(1), 75-97.

Lawson, R., Alcock, C., Cooper, J., \& Burgess, L. (2003). Factors affecting adoption of electronic commerce technologies by SMEs: an Australian study. Journal of Small Business and Enterprise Development, 10(3), 265-276.

Leonidou, L. C. (2004). An analysis of the barriers hindering small business export development. Journal of Small Business Management, 42(3), 279-302.

Lepoutre, J., \& Heene, A. (2006). Investigating the impact of firm size on small business social responsibility: A critical review. Journal of Business Ethics, 67(3), 257-273.

Levinthal, D. A., \& Warglien, M. (1999). Landscape design: Designing for local action in complex worlds. Organization Science, 10(3), 342357.

Lichtenthaler, U. (2011). Open innovation: Past research, current debates, and future directions. The Academy of Management Perspectives, 25(1), 75-93.

Madhok, A. (1996). Crossroads - the organization of economic activity: Transaction costs, firm capabilities, and the nature of governance. Organization Science, 7(5), 577-590.

Madrid-Guijarro, A., Garcia, D., \& Van Auken, H. (2009). Barriers to innovation among Spanish manufacturing SMEs. Journal of Small Business Management, 47(4), 465-488.

Mambula, C. (2002). Perceptions of SME growth constraints in Nigeria. Journal of Small Business Management, 40(1), 58.

March, J. G., \& Sutton, R. I. (1997). Crossroads—organizational performance as a dependent variable. Organization Science, 8(6), 698-706. Markides, C. (1998). Strategic innovation in established companies. Sloan Management Review, 39(3), 31. 
Matlay, H., \& Addis, M. (2003). Adoption of ICT and e-commerce in small businesses: an HEI-based consultancy perspective. Journal of Small Business and Enterprise Development, 10(3), 321-335.

Matthews, C. H., \& Moser, S. B. (1996). A longitudinal investigation of the impact of family background and gender on interest in small firm ownership. Journal of Small Business Management, 34(2), 29.

Michaelidou, N., Siamagka, N. T., \& Christodoulides, G. (2011). Usage, barriers and measurement of social media marketing: An exploratory investigation of small and medium B2B brands. Industrial Marketing Management, 40(7), 1153-1159.

Midgley, D. F. (1981). Toward a theory of the product life cycle: explaining diversity. The Journal of Marketing, 109-115.

Miettinen, R., \& Virkkunen, J. (2005). Epistemic objects, artefacts and organizational change. Organization, 12(3), 437-456.

Miles, R. E., Snow, C. C., Meyer, A. D., \& Coleman, H. J. (1978). Organizational strategy, structure, and process. Academy of Management Review, 3(3), 546-562.

Morris, M. H., Miyasaki, N. N., Watters, C. E., \& Coombes, S. M. (2006). The dilemma of growth: Understanding venture size choices of women entrepreneurs. Journal of Small Business Management, 44(2), 221-244.

Moy, J. W., \& Luk, V. W. (2003). The life cycle model as a framework for understanding barriers to SME growth in Hong Kong. Asia Pacific Business Review, 10(2), 199-220.

Murillo, D., \& Lozano, J. M. (2006). SMEs and CSR: An approach to CSR in their own words. Journal of Business Ethics, 67(3), 227-240.

Ohlott, P. J., Ruderman, M. N., \& McCauley, C. D. (1994). Gender differences in managers' developmental job experiences. Academy of Management Journal, 37(1), 46-67.

Olie, R. (1994). Shades of culture and institutions-in international mergers. Organization Studies, 15(3), 381-405.

Orser, B. J., Hogarth-Scott, S., \& Riding, A. L. (2000). Performance, firm size, and management problem solving. Journal of Small Business Management, 38(4), 42.

Park, S. H., \& Ungson, G. R. (2001). Interfirm rivalry and managerial complexity: A conceptual framework of alliance failure. Organization Science, 12(1), 37-53.

Perrini, F. (2006). SMEs and CSR theory: Evidence and implications from an Italian perspective. Journal of Business Ethics, 67(3), 305-316. Petersen, M. A., \& Rajan, R. G. (1994). The benefits of lending relationships: Evidence from small business data. The Journal of Finance, 49(1), 3-37.

Porter, M. E. (1998). Clusters and the new economics of competition (Vol. 76, No. 6, pp. 77-90). Boston: Harvard Business Review.

Ram, M., \& Smallbone, D. (2003). Policies to support ethnic minority enterprise: the English experience. Entrepreneurship \& Regional Development, 15(2), 151-166.

Repenning, N. P., \& Sterman, J. D. (2001). Nobody ever gets credit for fixing problems that never happened: creating and sustaining process improvement. California Management Review, 43(4), 64-88.

Robson, P. J., \& Obeng, B. A. (2008). The barriers to growth in Ghana. Small Business Economics, 30(4), 385-403.

Roth, A. V., Tsay, A. A., Pullman, M. E., \& Gray, J. V. (2008). Unraveling the food supply chain: strategic insights from China and the 2007 recalls. Journal of Supply Chain Management, 44(1), 22-39.

Roxas, B., Chadee, D., \& Erwee, R. (2012). Effects of rule of law on firm performance in South Africa. European Business Review, 24(5), 478-492.

Salvador, F., De Holan, P. M., \& Piller, F. T. (2009). Cracking the code of mass customization. MIT Sloan Management Review, 50 (3), 71.

Schefczyk, M. (1993). Operational performance of airlines: an extension of traditional measurement paradigms. Strategic Management Journal, 14(4), 301-317.

Schoemaker, P. J. (1993). Multiple scenario development: Its conceptual and behavioral foundation. Strategic Management Journal, 14(3), 193-213.

Sharpe, S. A. (1990). Asymmetric information, bank lending, and implicit contracts: A stylized model of customer relationships. The Journal of Finance, 45(4), 1069-1087.

Shepherd, D. A. (2015). Party On! A call for entrepreneurship research that is more interactive, activity based, cognitively hot, compassionate, and prosocial. Journal of Business Ventury, $30(4), 489-507$

Shimizu, K., \& Hitt, M. A. (2004). Strategic flexibility: Organizational preparedness to reverse ineffective strategic decisions. The Academy of Management Executive, 18(4), 44-59.

Smith-Hunter, A. E., \& Boyd, R. L. (2004). Applying theories of entrepreneurship to a comparative analysis of white and minority women business owners. Women in Management Review, 19(1), 18-28.

Sørensen, J. B., \& Stuart, T. E. (2000). Aging, obsolescence, and organizational innovation. Administrative Science Quarterly, 45(1), 81-112. Sørensen, J. B. (2002). The strength of corporate culture and the reliability of firm performance. Administrative Science Quarterly, 47(1), 70Storey, D. J. (1994). Understanding the small business sector. Routledge.

Strahan, P. E., \& Weston, J. P. (1998). Small business lending and the changing structure of the banking industry. Journal of Banking \& Finance, 22(6), 821-845.

Tabachnick, B. G., \& Fidel, L. S. (1983). Using multivariate statistics. New York: Harper \& Row.

Teng, J. T., Cheon, M. J., \& Grover, V. (1995). Decisions to outsource information systems functions: testing a strategy-theoretic discrepancy model. Decision Sciences, 26(1), 75-103.

Tetteh, E., \& Burn, J. (2001). Global strategies for SME-business: applying the small framework. Logistics Information Management, 14(1/2) Watson, J. (2012). Networking: Gender differences and the association with firm performance. International Small Business Journal, 30(5) Whetten, D. A. (2009). An examination of the interface between context and theory applied to the study of Chinese organizations. Management and Organization Review, 5(1), 29-55.

Vaara, E. (2003). Post-acquisition integration as sensemaking: Glimpses of ambiguity, confusion, hypocrisy, and politicization. Journal of Management Studies, 40(4), 859-894.

Von Bertalanffy, L. (1950). The theory of open systems in physics and biology. Science, 111(2872), 23-29.

Voss, G. B., Sirdeshmukh, D., \& Voss, Z. G. (2008). The effects of slack resources and environmentalthreat on product exploration and exploitation. Academy of Management Journal, 51(1), 147-164.

Yasuda, T. (2005). Firm growth, size, age and behavior in Japanese manufacturing. Small Business Economics, 24(1), 1-15. 